\title{
Complete minimal surfaces and harmonic functions
}

\author{
Antonio Alarcón*, Isabel Fernández** and Francisco J. López***
}

\begin{abstract}
We prove that for any open Riemann surface $\mathcal{N}$ and any non-constant harmonic function $h: \mathcal{N} \rightarrow \mathbb{R}$, there exists a complete conformal minimal immersion $X: \mathcal{N} \rightarrow \mathbb{R}^{3}$ whose third coordinate function coincides with $h$.

As a consequence, complete minimal surfaces with arbitrary conformal structure and whose Gauss map misses two points are constructed.
\end{abstract}

Mathematics Subject Classification (2010). 49Q05; 30F15, 53C42, 32H02.

Keywords. Complete minimal surfaces, harmonic functions on Riemann surfaces, Gauss map, holomorphic immersions.

\section{Introduction}

Conformal minimal immersions of Riemann surfaces in $\mathbb{R}^{3}$ are harmonic maps. This basic fact has strongly influenced the global theory of minimal surfaces, supplying this field with powerful tools coming from classical complex analysis and Riemann surfaces theory.

If $X=\left(X_{1}, X_{2}, X_{3}\right): \mathcal{N} \rightarrow \mathbb{R}^{3}$ is conformal and minimal, the holomorphic 1-forms $\phi_{j}:=\partial X_{j}, j=1,2,3$, satisfy the equation $\phi_{1}^{2}+\phi_{2}^{2}+\phi_{3}^{2}=0$. As a consequence, any conformal minimal immersion is uniquely determined (up to translations) by any two of its harmonic coordinate functions. On the other hand, it is reasonable to think that the family of conformal minimal immersions with $a$ prescribed coordinate function is in general vast. However, the construction of this kind of surfaces turns out to be more complicated than expected under completeness assumptions. A pioneering result in this direction can be found in $[\mathrm{AF}]$, where a

\footnotetext{
* Supported by Vicerrectorado de Política Científica e Investigación de la Universidad de Granada. Research partially supported by MCYT-FEDER research projects MTM2007-61775 and MTM2011-22547, Junta de Andalucía Grant P09-FQM-5088, and the grant PYR-2012-3 CEI BioTIC GENIL (CEB09-0010) of the MICINN CEI Program.

**Research partially supported by MCYT-FEDER research project MTM2010-19821 and Junta de Andalucía Grant P09-FQM-5088.

*** Research partially supported by MCYT-FEDER research projects MTM2007-61775 and MTM2011-22547, and Junta de Andalucía Grant P09-FQM-5088.
} 
satisfactory answer in the simply connected case is given. The aim of this paper is to extend this result to the more general setting of arbitrary open Riemann surfaces.

Our main theorem asserts that:

Theorem I. Let $\mathcal{N}$ be an open Riemann surface, let $h: \mathcal{N} \rightarrow \mathbb{R}$ be a non-constant harmonic function and let $p: \mathscr{H}_{1}(\mathcal{N}, \mathbb{Z}) \rightarrow \mathbb{R}^{3}$ be a group morphism such that the third coordinate of $p(\gamma)$ coincides with $\operatorname{Im} \int_{\gamma} \partial h$, for all $\gamma \in \mathscr{H}_{1}(\mathcal{N}, \mathbb{Z})$.

Then there exists a complete conformal minimal immersion

$$
X=\left(X_{1}, X_{2}, X_{3}\right): \mathcal{N} \rightarrow \mathbb{R}^{3}
$$

with $X_{3}=h$ and flux map $p_{X}=p$.

Recall that the flux map of a conformal minimal immersion $X: \mathcal{N} \rightarrow \mathbb{R}^{3}$ is given by $p_{X}(\gamma)=\operatorname{Im} \int_{\gamma} \partial X$, for all $\gamma \in \mathscr{H}_{1}(\mathcal{N}, \mathbb{Z})$.

As a consequence of Theorem I, we obtain some interesting results concerning the Gauss map of minimal surfaces, the Calabi-Yau problem, holomorphic null curves in $\mathbb{C}^{3}$ and maximal surfaces in the Lorentz-Minkowski space $\mathbb{R}_{1}^{3}$.

The study of the Gauss map is one of the fundamental problems in the theory of minimal surfaces. Fujimoto $[\mathrm{Fu}]$ showed that the number of exceptional values of the Gaussian image of a complete non-flat minimal surface is at most four, improving some classical results by Osserman [Os1] and Xavier [Xa]. Since Sherk's minimal surfaces omit four points, then Fujimoto's theorem is sharp. However, the number of exceptional values strongly depends on the underlying conformal structure. For instance, by Picard's theorem there are no conformal non-flat minimal immersions of the complex plane in $\mathbb{R}^{3}$ whose Gauss map omits three points. So it is natural to wonder whether any open Riemann surface admits a complete conformal minimal immersion with Gauss map omitting two points. We answer affirmatively this question, proving considerably more:

Theorem II. Let $\mathcal{N}$ be an open Riemann surface, and let $p: \mathscr{H}_{1}(\mathcal{N}, \mathbb{Z}) \rightarrow \mathbb{R}^{3}$ be a group morphism.

Then there exists a complete conformal minimal immersion $X: \mathcal{N} \rightarrow \mathbb{R}^{3}$ whose Gauss map omits two antipodal points and $p_{X}=p$.

Calabi-Yau conjectures deal with the existence problem of complete minimal surfaces with bounded coordinate functions. There is large literature on this topic, see $[\mathrm{JX}],[\mathrm{Na}],[\mathrm{CM}],[\mathrm{FMM}]$ for a good setting. From Theorem I follows that a (necessary and) sufficient condition for an open Riemann surface to admit a complete conformal non-flat minimal immersion into an open slab of $\mathbb{R}^{3}$ is to carry non-constant bounded harmonic functions (see Corollary 4.3).

Likewise, by Theorem I, if $\mathcal{N}$ is an open Riemann surface and $f: \mathcal{N} \rightarrow \mathbb{C}$ a nonconstant holomorphic function, there exists a complete null holomorphic immersion $\left(F_{1}, F_{2}, F_{3}\right): \mathcal{N} \rightarrow \mathbb{C}^{3}$ (and so a complete holomorphic immersion $\left(F_{1}, F_{3}\right): \mathcal{N} \rightarrow$ $\mathbb{C}^{2}$ ) with $F_{3}=f$. The family of open Riemann surfaces admitting non-constant 
bounded holomorphic functions is particularly interesting from several points of view. This space contains examples of arbitrary open topological type, and as above any such surface admits a complete null holomorphic immersion in $\mathbb{C}^{2} \times \mathbb{D}$ (and so a complete holomorphic immersion in $\mathbb{C} \times \mathbb{D}$ ). We have compiled these ideas in the following result (for the construction of proper complete null curves in $\mathbb{C}^{2} \times \mathbb{D}$ and proper complete holomorphic curves in $\mathbb{C} \times \mathbb{D}$ see Corollary 4.4):

Corollary III. Let $M$ be an open orientable surface. Then there exists a complete minimal surface homeomorphic to $M$ all whose associate surfaces are well defined and contained in a slab of $\mathbb{R}^{3}$.

Complete minimal surfaces properly immersed in an open slab of $\mathbb{R}^{3}$ of arbitrary topological type can be found in [FMM] (see also [JX], [RT], [Lo1], [Lo2], [MM], [AFM] for a good setting). The problem of constructing bounded complete null holomorphic curves in $\mathbb{C}^{3}$ has been solved in [AL2].

Finally, Theorem I provides weakly complete conformal maximal immersions in the Lorentz-Minkowski 3-spacetime $\mathbb{R}_{1}^{3}$ with singularities and prescribed spacelike or timelike coordinate functions (the notion of weakly complete maximal surface with singularities was defined in [UY]). See Corollary 4.6 for more details.

In a forthcoming paper [AL2], the authors will extend these results to the nonorientable setting.

\section{Preliminaries}

For a topological surface $M$, we will denote as $\partial(M)$ the one dimensional topological manifold determined by the boundary points of $M$. Given $S \subset M, S^{\circ}$ and $\bar{S}$ will denote the interior and the closure of $S$ in $M$, respectively. A Riemann surface $M$ is said to be open if it is non-compact and $\partial(M)=\emptyset$.

Remark 2.1. In the sequel $\mathcal{N}$ will denote a fixed but arbitrary open Riemann surface, $W \subset \mathcal{N}$ an open connected subset of finite topology, and $S \subset W$ a compact set.

For a proper subset $M$ of $\mathcal{N}$ we will denote by $\Omega_{0}(M)$ as the space of holomorphic 1 -forms on an open neighborhood of $S$ in $\mathcal{N}$, whereas $\Omega_{0}^{*}(M)$ will denote the space of complex 1-forms $\theta$ of type $(1,0)$ that are continuous on $M$ and holomorphic on $M^{\circ}$. As usual, a 1 -form $\theta$ on $M$ is said to be of type $(1,0)$ if for any conformal chart $(U, z)$ in $\mathcal{N},\left.\theta\right|_{U \cap M}=h(z) d z$ for some function $h: U \cap M \rightarrow \mathbb{C}$.

Definition 2.2 (Admissible set). A compact subset $S \subset W$ is said to be admissible in $W$ if and only if:

- $W-S$ has no bounded components in $W$ (by definition, a connected component $V$ of $W-S$ is said to be bounded in $W$ if $\bar{V} \cap W$ is compact, where $\bar{V}$ is the closure of $V$ in $\mathcal{N}$ ), 
- $M_{S}:=\overline{S^{\circ}}$ consists of a finite collection of pairwise disjoint compact regions in $W$ with $\mathcal{C}^{0}$ boundary,

- $C_{S}:=\overline{S-M_{S}}$ consists of a finite collection of pairwise disjoint analytical Jordan arcs (recall that a compact Jordan arc in $\mathcal{N}$ is said to be analytical if it is contained in an open analytical Jordan arc in $\mathcal{N}$ ), and

- any component $\alpha$ of $C_{S}$ with an endpoint $P \in M_{S}$ admits an analytical extension $\beta$ in $W$ such that the unique component of $\beta-\alpha$ with endpoint $P$ lies in $M_{S}$.

Observe that if $S$ is admissible in $\mathcal{N}$ then it is admissible in $W$ as well, but the contrary is in general false.

With the previous notation, a function $f: S \rightarrow \mathbb{C}$ defined on an admissible set $S$ in $W$ is said to be smooth if $\left.f\right|_{M_{S}}$ admits a smooth extension $f_{0}$ to an open domain $V \subset W$ containing $M_{S}$, and for any component $\alpha$ of $C_{S}$ and any open analytical Jordan arc $\beta$ in $W$ containing $\alpha, f$ admits an smooth extension $f_{\beta}$ to $\beta$ satisfying that $\left.f_{\beta}\right|_{V \cap \beta}=\left.f_{0}\right|_{V \cap \beta}$.

Likewise, a 1-form $\theta \in \Omega_{0}^{*}(S)$ is said to be smooth if, for any closed conformal disk $(U, z)$ on $W$ such that $S \cap U$ is admissible in $W, \theta / d z$ is smooth in the previous sense.

Given a smooth function $f: S \rightarrow \mathbb{C}$ holomorphic on $S^{\circ}$, we set $d f \in \Omega_{0}^{*}(S)$ as the smooth 1-form given by $\left.d f\right|_{M_{S}}=d\left(\left.f\right|_{M_{S}}\right)$ and $\left.d f\right|_{\alpha \cap U}=\left.(f \circ \alpha)^{\prime}(x) d z\right|_{\alpha \cap U}$, where $(U, z=x+i y)$ is a conformal chart on $W$ such that $\alpha \cap U=z^{-1}(\mathbb{R} \cap z(U))$. Obviously, $\left.d f\right|_{\alpha}(t)=(f \circ \alpha)^{\prime}(t) d t$ for any component $\alpha$ of $C_{S}$, where $t$ is any smooth parameter along $\alpha$. A smooth 1-form $\theta \in \Omega_{0}^{*}(S)$ is said to be exact if $\theta=d f$ for some smooth $f: S \rightarrow \mathbb{C}$ holomorphic on $S^{\circ}$, or equivalently if $\int_{\gamma} \theta=0$ for all $\gamma \in \mathscr{H}_{1}(S, \mathbb{Z})$.

The following lemma and its corollaries will be required to approximate minimal immersions by immersions defined on larger domains (possibly with higher topology).

Lemma 2.3 ([AL], Approximation Lemma). Let $S$ be an admissible compact set in $W$, and $\Phi=\left(\phi_{j}\right)_{j=1,2,3}$ a smooth triple in $\Omega_{0}^{*}(S)^{3}$, such that $\sum_{j=1}^{3} \phi_{j}^{2}=0$, $\sum_{j=1}^{3}\left|\phi_{j}\right|^{2}$ never vanishes on $S$, and $\left.\Phi\right|_{M_{S}} \in \Omega_{0}\left(M_{S}\right)^{3}$.

Then it is possible to uniformly approximate $\Phi$ on $S$ by a sequence $\left\{\Phi_{n}=\right.$ $\left.\left(\phi_{j, n}\right)_{j=1,2,3}\right\}_{n \in \mathbb{N}}$ in $\Omega_{0}(W)^{3}$ satisfying

(i) $\sum_{j=1}^{3} \phi_{j, n}^{2}=0$,

(ii) $\sum_{j=1}^{3}\left|\phi_{j, n}\right|^{2}$ never vanishes on $W$ and

(iii) $\Phi_{n}-\Phi$ is exact on $S$, for all $n \in \mathbb{N}$.

Recall that a 1-form $\theta \in \Omega_{0}^{*}(S)$ is said to be uniformly approximated on $S$ by 1 -forms in $\Omega_{0}(W)$, if there exists $\left\{\theta_{n}\right\}_{n \in \mathbb{N}} \subset \Omega_{0}(W)$ such that $\left\{\frac{\theta_{n}-\theta}{d z}\right\}_{n \in \mathbb{N}} \rightarrow 0$ uniformly on $S \cap U$, for any conformal closed disc $(U, d z)$ on $W$. 
Corollary 2.4 ([AL], Corollary 4.8). The sequence $\left\{\Phi_{n}=\left(\phi_{j, n}\right)_{j=1,2,3}\right\}_{n \in \mathbb{N}}$ in the above lemma can be obtained such that $\phi_{3, n}=\phi_{3}$ for all $n \in \mathbb{N}$, provided that $\phi_{3}$ extends holomorphically to $W$ and never vanishes on $C_{S}$.

Corollary 2.5. The sequence $\left\{\Phi_{n}=\left(\phi_{j, n}\right)_{j=1,2,3}\right\}_{n \in \mathbb{N}}$ obtained in Lemma 2.3 can be chosen such that $\phi_{3, n}$ never vanishes on $W$, for all $n \in \mathbb{N}$, provided that $\phi_{3}$ never vanishes on $S$.

Remark 2.6. Although Corollary 2.5 is not explicitly stated in [AL], it can be deduced from the proof of the Approximation Lemma in [AL]. Indeed, the 1-form $\phi_{3, n}$ is defined as $\phi_{3, n}=e^{f_{n}} \psi_{n}$, where $f_{n}$ is a holomorphic function on $W$, and $\psi_{n} \in$ $\Omega_{0}(W)$ never vanishes on $W$ provided that $\phi_{3}$ does in $S, n \in \mathbb{N}$.

2.1. Minimal surfaces. As remarked in Section 1, the coordinates functions of a conformal minimal immersion $X=\left(X_{1}, X_{2}, X_{3}\right): W \rightarrow \mathbb{R}^{3}$ are harmonic. If we denote $\partial$ as the global complex operator given by $\left.\partial\right|_{U}=\frac{\partial}{\partial z} d z$ for any conformal chart $(U, z)$ on $W$, then the corresponding 1-forms $\phi_{j}=\partial X_{j}, j=1,2,3$, are holomorphic on $W$. Moreover, $X$ and its pull-back metric are given by

$$
X=\operatorname{Re} \int\left(\phi_{1}, \phi_{2}, \phi_{3}\right),
$$

and

$$
d s_{X}^{2}=\sum_{k=1}^{3}\left|\phi_{k}\right|^{2}
$$

respectively. As a consequence, the triple $\Phi=\left(\phi_{1}, \phi_{2}, \phi_{3}\right)$ satisfies the following properties:

(i) $\phi_{k}$ have no real periods, $k=1,2,3$,

(ii) $\sum_{k=1}^{3} \phi_{k}^{2}=0$,

(iii) $\phi_{k}, k=1,2,3$, have no common zeroes.

Conversely, given a vectorial holomorphic 1-form $\Phi=\left(\phi_{1}, \phi_{2}, \phi_{3}\right)$ on $W$ satisfying (i) to (iii), then (2.1) determines a conformal minimal immersion $X: W \rightarrow \mathbb{R}^{3}$.

The triple $\Phi$ is said to be the Weierstrass representation of $X$. A remarkable fact is that the stereographic projection of the Gauss map of $X$ is the (meromorphic) function $g=\frac{\phi_{3}}{\phi_{1}-i \phi_{2}}$. In particular, the poles and zeros of $g$ coincide with the zeros of $\phi_{3}$ with the same order (see [Os2]).

The flux of $X$ along a closed curve $\gamma$ in $W$ is defined as $p_{X}(\gamma)=\int_{\gamma} \mu(s) d s$, where $s$ is the arclength parameter of $\gamma$ and $\mu(s)$ is the conormal vector of $X$ at $\gamma(s)$ (i.e., the unique vector such that $\left\{d X\left(\gamma^{\prime}(s)\right), \mu(s)\right\}$ is an orthonormal positive basis of the tangent plane of $X$ at $\gamma(s))$. It is easy to check that $p_{X}(\gamma)=\operatorname{Im} \int_{\gamma} \partial X$ and that the flux map $p_{X}: \mathscr{H}_{1}(M, \mathbb{Z}) \rightarrow \mathbb{R}^{3}$ is a group morphism. 
As we will deal with admissible sets, a suitable notion for minimal immersions on admissible sets will be required. This is the aim of the following definitions.

Let $S$ be a admissible subset in $W$ and $X: S \rightarrow \mathbb{R}^{3}$ a smooth map such that $\left.X\right|_{C_{S}}$ is regular, (i.e., $\left.X\right|_{\alpha}$ is a regular curve for all $\alpha \subset C_{S}$ ). By a smooth normal field along $C_{S}$ respect to $X$ we mean a field $\varpi: C_{S} \rightarrow \mathbb{R}^{3}$ such that, for any analytical arc $\alpha \subset C_{S}, \varpi \circ \alpha$ is smooth, unitary and orthogonal to $(X \circ \alpha)^{\prime}, \varpi$ extends smoothly to any open analytical arc $\beta$ in $W$ containing $\alpha$, and $\varpi$ is tangent to $X$ on $\beta \cap S$. The normal field $\varpi$ is said to be orientable respect to $X$ if for any component $\alpha \subset C_{S}$ with endpoints $P_{1}, P_{2} \in \partial\left(M_{S}\right)$, and for any arclength parameter $s$ along $\left.X\right|_{\alpha}$, the basis $B_{i}=\left\{\left(\left.X\right|_{\alpha}\right)^{\prime}\left(s_{i}\right), \varpi\left(s_{i}\right)\right\}$ of the tangent plane of $\left.X\right|_{M_{S}}$ at $P_{i}, i=1,2$, are both positive or negative, where $s_{i}$ is the value of $s$ for which $\alpha\left(s_{i}\right)=P_{i}, i=1,2$.

Definition 2.7. Given a proper subset $M \subset \mathcal{N}$, we denote by $\mathcal{M}(M)$ the space of maps $X: M \rightarrow \mathbb{R}^{3}$ extending as a conformal minimal immersion to an open neighborhood of $M$ in $\mathcal{N}$. On the other hand, for an admissible set $S$ in $W$ we call $\mathcal{M}^{*}(S)$ as the space of marked immersions $X_{\varpi}:=(X, \varpi)$, where

(1) $X: S \rightarrow \mathbb{R}^{3}$ is a smooth map,

(2) $\left.X\right|_{M_{S}} \in \mathcal{M}\left(M_{S}\right)$,

(3) $\left.X\right|_{C_{S}}$ is regular, and

(4) $\varpi$ is an orientable smooth normal field along $C_{S}$ respect to $X$.

We will endow $\mathcal{M}(M)$ (resp. $\mathcal{M}^{*}(S)$ ) with the $\mathcal{C}^{0}$ topology of the uniform convergence on compact subsets of $M$ (resp. uniform convergence of maps and normal fields on $S$ ).

The notions of Weierstrass data and flux map can be also extended to immersions in $\mathcal{M}^{*}(S)$. Indeed, given $X_{\varpi} \in \mathcal{M}^{*}(S)$, let $\partial X_{\varpi}=\left(\hat{\phi}_{j}\right)_{j=1,2,3}$ be the complex vectorial 1-form on $S$ given by $\partial X_{\varpi}:=\partial\left(\left.X\right|_{M_{S}}\right)$, and for any component $\alpha$ of $C_{S}$, $\partial X_{\varpi}:=d X\left(\alpha^{\prime}(s)\right)+i \varpi(s)$, where $s$ is the arclength parameter of $\left.X\right|_{\alpha}$ such that $\left\{d X\left(\alpha^{\prime}\left(s_{0}\right)\right), \varpi\left(s_{0}\right)\right\}$ is positive provided that $\alpha\left(s_{0}\right) \in \partial\left(M_{S}\right)$.

The triple $\widehat{\Phi}:=\partial X_{\varpi}$ will be called the generalized Weierstrass data of $X_{\varpi}$. It is clear that $\widehat{\Phi} \in \Omega_{0}^{*}(S)^{3}$ and is smooth. Notice also that $\sum_{j=1}^{3} \hat{\phi}_{j}^{2}=0, \sum_{j=1}^{3}\left|\hat{\phi}_{j}\right|^{2}$ never vanishes on $S$ and $\operatorname{Real}\left(\hat{\phi}_{j}\right)$ is an exact real 1 -form on $S, j=1,2,3$, hence we also have $X(P)=X(Q)+\operatorname{Real} \int_{Q}^{P}\left(\hat{\phi}_{j}\right)_{j=1,2,3}, P, Q \in S$. In particular, since $\left.X\right|_{M_{S}} \in \mathcal{M}\left(M_{S}\right)$ then $\left(\phi_{j}\right)_{j=1,2,3}:=\left(\left.\hat{\phi}_{j}\right|_{M_{S}}\right)_{j=1,2,3}$ are the Weierstrass data of $\left.X\right|_{M_{S}}$.

The group homomorphism

$$
p_{X_{\varpi}}: \mathscr{H}_{1}(S, \mathbb{Z}) \rightarrow \mathbb{R}^{3}, \quad p_{X_{\varpi}}(\gamma)=\operatorname{Im} \int_{\gamma} \partial X_{\varpi},
$$


is said to be the generalized flux map of $X_{\varpi}$. Obviously, $p_{X_{\varpi_{Y}}}=\left.p_{Y}\right|_{\mathscr{H}_{1}(S, \mathbb{Z})}$ provided that $X=\left.Y\right|_{S}$ and $\varpi_{Y}$ is the conormal field of $Y \in \mathcal{M}(W)$ along any curve in $C_{S}$.

\section{The completeness lemma}

Given a compact subset $M \subset \mathcal{N}$ and a map $X=\left(X_{1}, X_{2}, X_{3}\right): M \rightarrow \mathbb{R}^{3}$, we denote $\|X\|:=\max _{M}\left\{\left(\sum_{j=1}^{3} X_{j}^{2}\right)^{1 / 2}\right\}$ as the maximum norm of $X$ on $M$.

The following lemma concentrates most of the technical computations required in the proof of the main result of this paper.

Lemma 3.1. Let $U, V$ be two compact regions in $\mathcal{N}$ such that $U \subset V^{\circ}$ and $V^{\circ}-U$ has no bounded components in $V^{\circ}$. Consider a non-constant harmonic function $h: V \rightarrow \mathbb{R}$, an immersion $X=\left(X_{1}, X_{2}, X_{3}\right) \in \mathcal{M}(U)$ and a group morphism $p: \mathscr{H}_{1}(V, \mathbb{Z}) \rightarrow \mathbb{R}^{3}$ such that $X_{3}=\left.h\right|_{U}, p_{X}=\left.p\right|_{\mathscr{H}_{1}(U, \mathbb{Z})}$ and the third coordinate of $p(\gamma)$ is $\operatorname{Im} \int_{\gamma} \partial h$, for all $\gamma \in \mathscr{H}_{1}(V, \mathbb{Z})$.

Then, for any $P_{0} \in U$ and $\epsilon>0$, there exists $Y=\left(Y_{1}, Y_{2}, Y_{3}\right) \in \mathcal{M}(V)$ satisfying the following:

(i) $\|Y-X\|<\epsilon$ on $U$,

(ii) $Y_{3}=h$,

(iii) $p_{Y}=p$ and

(iv) $\operatorname{dist}_{Y}\left(P_{0}, \partial(V)\right)>1 / \epsilon$.

Here dist $_{Y}$ denotes the distance on $V$ in the intrinsic metric of the immersion $Y$.

Proof. We will prove this lemma by induction on (minus) the Euler characteristic of $V^{\circ}-U$ (recall that, since we are assuming that $V^{\circ}-U$ has no bounded components in $V^{\circ}$, then $\left.\chi\left(V^{\circ}-U\right) \leq 0\right)$. The induction process is enclosed in the following two claims.

Claim 3.2. The lemma holds if $\chi\left(V^{\circ}-U\right)=0$.

Proof. The argument we use now is analogous to the one employed in Lemma 1 of [AF]. Write $V^{\circ}-U=\bigcup_{j=1}^{k} A_{j}$, where $A_{j}$ are pairwise disjoint open annuli. On each component $A_{j}$ we define the following labyrinth of compact sets. Let $z_{j}: A_{j} \rightarrow \mathbb{C}$ be a conformal parametrization, and consider a compact region $C_{j} \subset A_{j}$ such that $C_{j}$ contains no zeros of $\partial h, z_{j}\left(C_{j}\right)$ is a compact annulus of radii $r_{j}$ and $R_{j}$, where $r_{j}<$ $R_{j}$, and $z_{j}\left(C_{j}\right)$ contains the homology of $z_{j}\left(A_{j}\right)$. Write $\phi_{3}=\partial X_{3}=f_{j}\left(z_{j}\right) d z_{j}$, with $\left|f_{j}\right|>0$ on $C_{j}$. Let $\mu$ be a positive constant with

$$
\mu<\min \left\{\left|f_{j}(P)\right| \mid P \in C_{j}, j=1, \ldots, k\right\} .
$$


Fix a natural number $N$ (to be specified later) such that $2 / N<\min \left\{R_{j}-r_{j} \mid j=\right.$ $1, \ldots, k\}$. For any $n \in\left\{1, \ldots, 2 N^{2}\right\}$, consider the compact set in $C_{j}$ :

$$
\begin{array}{r}
\mathcal{K}_{j, n}=\left\{p \in A_{j}\left|s_{n}+\frac{1}{4 N^{3}} \leq\right| z_{j}(p) \mid \leq s_{n-1}-\frac{1}{4 N^{3}},\right. \\
\left.\frac{1}{N^{2}} \leq \arg \left((-1)^{n} z_{j}(p)\right) \leq 2 \pi-\frac{1}{N^{2}}\right\},
\end{array}
$$

where $s_{n}:=R_{j}-n / N^{3}$. Then, define

$$
\mathcal{K}_{j}=\bigcup_{n=1}^{2 N^{2}} \mathcal{K}_{j, n} \quad \text { and } \quad \mathcal{K}=\bigcup_{j=1}^{k} \mathcal{K}_{j}
$$

Define $\Phi \in \Omega_{0}(U \cup \mathcal{K})^{3}$ by

$$
\left.\Phi\right|_{U}=\partial X,\left.\quad \Phi\right|_{\mathcal{K}}=\left(\frac{1}{2}\left(\frac{1}{M}-M\right) \phi_{3}, \frac{i}{2}\left(\frac{1}{M}+M\right) \phi_{3}, \phi_{3}\right),
$$

where $M>2 N^{4}$ is a constant.

By Corollary 2.4 applied to $S=U \cup \mathcal{K}, \Phi$, and an open tubular neighborhood of $V$, we can infer the existence of $\Psi \in \Omega_{0}(V)^{3}$ giving rise to a well-defined conformal minimal immersion $Y=\left(Y_{1}, Y_{2}, Y_{3}\right) \in \mathcal{M}(V)$ fulfilling (i), (ii) and (iii), and whose metric $d s_{Y}^{2}$ satisfies

$$
d s_{Y}^{2}>\frac{1}{4}\left(\frac{1}{M}+M\right)^{2} \mu^{2}\left|d z_{j}\right|^{2}>N^{8} \mu^{2}\left|d z_{j}\right|^{2} \quad \text { on } \mathcal{K}_{j}, j=1, \ldots, k .
$$

To finish the claim it remains to check (iv). Taking into account that $d s_{Y}^{2} \geq$ $\left|\phi_{3}\right|^{2}>\mu^{2}\left|d z_{j}\right|^{2}$ on $C_{j}$, and (3.1), it is not hard to check that there exists a positive constant $\rho_{j}$ depending neither on $\mu$ nor $N$ such that

$$
\text { length }_{d s_{Y}^{2}}(\alpha)>\rho_{j} \cdot \mu \cdot N
$$

for any $\alpha$ curve in $C_{j}$ joining the two components of $\partial\left(C_{j}\right)$. Thus, we can choose $N$ large enough such that $\rho_{j} \cdot \mu \cdot N>1 / \epsilon$ for any $j=1, \ldots, k$. In particular, (iv) is achieved.

Claim 3.3. Let $n>0$. Assume that the lemma holds if $-\chi\left(V^{\circ}-U\right)<n$. Then it also holds for $-\chi\left(V^{\circ}-U\right)=n$.

Proof. Since $-\chi\left(V^{\circ}-U\right)>0$, there exists $\hat{\gamma} \in \mathscr{H}_{1}(V, \mathbb{Z})-\mathscr{H}_{1}(U, \mathbb{Z})$ intersecting $V^{\circ}-U^{\circ}$ in a Jordan arc $\gamma$ with endpoints $P_{1}, P_{2} \in \partial(U)$ and otherwise disjoint from $\partial(U)$, and such that $S:=U \cup \gamma$ is an admissible set in an open tubular neighborhood $W$ of $V$ in $\mathcal{N}$. Moreover, we take $\hat{\gamma}$ such that $\partial h$ never vanishes on $\gamma$. 
Take $F_{\varpi} \in \mathcal{M}^{*}(S), F=\left(F_{1}, F_{2}, F_{3}\right)$, satisfying $\left.F\right|_{U}=X, F_{3}=\left.h\right|_{S}$, the third coordinate of $\partial F_{\varpi}$ is $\left.\partial h\right|_{S}$, and $p_{F_{\varpi}}(\hat{\gamma})=p(\hat{\gamma})$.

By Corollary 2.4 applied to the (generalized) Weierstrass data of $F_{\varpi}, S$ and $W$, we obtain a compact tubular neighborhood $W^{\prime}$ of $S$ in $V^{\circ}$ and $Z=\left(Z_{1}, Z_{2}, Z_{3}\right) \in$ $\mathcal{M}\left(W^{\prime}\right)$ such that $\|Z-X\|<\epsilon / 2$ on $U, p_{Z}=\left.p\right|_{\mathscr{H}_{1}\left(W^{\prime}, \mathbb{Z}\right)}$, and $Z_{3}=\left.h\right|_{W^{\prime}}$. Since $-\chi\left(V^{\circ}-W^{\prime}\right)<n$, the induction hypothesis applied to $Z$ and $\epsilon / 2$ gives the existence of an immersion $Y$ satisfying the conclusion of the lemma.

The proof is done.

\section{Main results}

In this section we prove the results stated in the introduction and obtain some corollaries.

Theorem 4.1. Let $h: \mathcal{N} \rightarrow \mathbb{R}$ be a non-constant harmonic function and let $p: \mathscr{H}_{1}(\mathcal{N}, \mathbb{Z}) \rightarrow \mathbb{R}^{3}$ be a group morphism such that the third coordinate of $p(\gamma)$ coincides with $\operatorname{Im} \int_{\gamma} \partial h$, for all $\gamma \in \mathscr{H}_{1}(\mathcal{N}, \mathbb{Z})$.

Then there exists a complete conformal minimal immersion

$$
X=\left(X_{1}, X_{2}, X_{3}\right): \mathcal{N} \rightarrow \mathbb{R}^{3}
$$

with $X_{3}=h$ and $p_{X}=p$.

Proof. Consider an exhaustive sequence $\left\{V_{n}\right\}_{n \in \mathbb{N}} \subset \mathcal{N}$ of compact regions such that $V_{1}$ is simply connected, $V_{n-1} \subset V_{n}^{\circ}$, and $V_{n}^{\circ}-V_{n-1}$ has no bounded components in $V_{n}^{\circ}, n \geq 2$.

Let $Y_{1} \in \mathcal{M}\left(V_{1}\right)$ be the conformal minimal immersion with Weierstrass data given by $\phi_{3}=\left.(\partial h)\right|_{V_{1}}$ and $g=\phi_{3} / d z$, where $z$ is a conformal parameter on $V_{1}$.

Fix a point $P_{0} \in V_{1}^{\circ}$, and apply recursively Lemma 3.1 to obtain a sequence $\left\{Y_{n}\right\}_{n \in \mathbb{N}}, Y_{n} \in \mathcal{M}\left(V_{n}\right)$ satisfying that:

a) $\left\|Y_{n}-Y_{n-1}\right\|<1 / n^{2}$ on $V_{n-1}$,

b) $\operatorname{dist}_{Y_{n}}\left(P_{0}, \partial\left(V_{n}\right)\right)>n^{2}$,

c) $p_{Y_{n}}=p \mid \mathscr{H}_{1}\left(V_{n}, \mathbb{Z}\right)$, and

d) the third coordinate function of $Y_{n}$ coincides with $\left.h\right|_{V_{n}}$,

for all $n \in \mathbb{N}$. Here $\operatorname{dist}_{Y_{n}}$ denotes the distance on $V_{n}$ in the intrinsic metric of the immersion $Y_{n}$. Since $\mathcal{N}=\bigcup_{n \in \mathbb{N}} V_{n}$, property a) gives that $\left\{Y_{n}\right\}_{n \in \mathbb{N}}$ converges to a harmonic limit map $X=\left(X_{1}, X_{2}, X_{3}\right): \mathcal{N} \rightarrow \mathbb{R}^{3}$ uniformly on compact sets (Harnack's theorem). Moreover, from Hurwitz' theorem and the fact that $\partial Y_{n}$ never vanishes we infer that either $X$ degenerates on a point or has no branch points. 
From d) follows $X_{3}=h$ which is non-constant and so the first possibility can not occur. On the other hand, properties b) and c) give that $X$ is complete and $p_{X}=p$, respectively.

Any open Riemann surface carries regular harmonic functions, that is to say, harmonic functions with never vanishing differential. As a consequence, any open Riemann surface admits a conformal complete minimal immersion in $\mathbb{R}^{3}$ whose Gauss map misses two antipodal values. For completeness we include a detailed proof of all these facts based in Corollary 2.5.

Theorem 4.2. Let $p: \mathscr{H}_{1}(\mathcal{N}, \mathbb{Z}) \rightarrow \mathbb{R}^{3}$ be a group morphism.

Then there exists a complete conformal minimal immersion $X: \mathcal{N} \rightarrow \mathbb{R}^{3}$ such that its meromorphic Gauss map has neither zeros nor poles and $p_{X}=p$.

Proof. Take $\left\{V_{n}\right\}_{n \in \mathbb{N}} \subset \mathcal{N}$ an exhaustive sequence of compact regions such that $V_{1}$ is simply connected, $V_{n} \subset V_{n+1}^{\circ}, V_{n+1}^{\circ}-V_{n}$ has no bounded components and $\chi\left(V_{n+1}^{\circ}-V_{n}\right)=-1$. Let $F \in \mathcal{M}\left(V_{1}\right)$ be a conformal minimal immersion with Weierstrass data $\Psi=\left(\psi_{1}, \psi_{2}, \psi_{3}\right)$ such that $\psi_{3}$ never vanishes on $V_{1}$.

Fix $\epsilon>0$. The key step in the proof is the construction of a sequence $\left\{Y_{n}\right\}_{n \in \mathbb{N}}$, $Y_{n} \in \mathcal{M}\left(V_{n}\right)$ with Weierstrass data $\Phi_{n}=\left\{\left(\phi_{j, n}\right)_{j=1,2,3}\right\}$ satisfying that:

a) $\left\|Y_{n}-Y_{n-1}\right\|<\epsilon / n^{2}$ on $V_{n-1}$,

b) $p_{Y_{n}}=\left.p\right|_{\mathscr{H}_{1}\left(V_{n}, \mathbb{Z}\right)}$ and

c) $\phi_{3, n}$ never vanishes on $V_{n}$,

for all $n \geq 2$.

Indeed, choose $Y_{1}=F$ and assume that we have constructed $Y_{1}, \ldots, Y_{n}$. Then the immersion $Y_{n+1}$ is defined as follows. Let $\hat{\gamma} \in \mathscr{H}_{1}\left(V_{n+1}, \mathbb{Z}\right)-\mathscr{H}_{1}\left(V_{n}, \mathbb{Z}\right)$ intersecting $V_{n+1}-V_{n}^{\circ}$ in a Jordan arc $\gamma$ with endpoints $P_{1}, P_{2} \in \partial\left(V_{n}\right)$ and otherwise disjoint from $\partial\left(V_{n}\right)$, and such that $S:=V_{n} \cup \gamma$ is an admissible set in an open tubular neighborhood $W$ of $V_{n+1}$ in $\mathcal{N}$. Then extend $Y_{n}$ to a marked immersion $Z_{\varpi} \in \mathcal{M}^{*}(S)$ satisfying that $p_{Z_{\varpi}}=\left.p\right|_{\mathscr{H}_{1}(S, \mathbb{Z})}$ and the third coordinate of $\partial Z_{\varpi}$ never vanishes on $\gamma$. Applying Corollary 2.5 to the generalized Weierstrass data of $Z_{\varpi}, S$ and $W$, and integrating the resulting 1-forms we get $Y_{n+1} \in \mathcal{M}\left(V_{n+1}\right)$ satisfying the desired conditions.

By a), Harnack's theorem and Hurwitz' theorem, the sequence $\left\{Y_{n}\right\}_{n \in \mathbb{N}}$ converges uniformly on compact sets to a conformal minimal immersion $Y: \mathcal{N} \rightarrow \mathbb{R}^{3}$, provided that $\epsilon$ is small enough. Label $\Phi=\left(\phi_{1}, \phi_{2}, \phi_{3}\right)$ as its Weierstrass data. It is clear that $p=p_{Y}$, let us check now that $\phi_{3}$ never vanishes. Indeed, assume $\phi_{3}$ has a zero at a point in $V_{n_{0}}$, for $n_{0} \in \mathbb{N}$. Since $\phi_{3, n}$ never vanishes in $V_{n_{0}}$ for all $n \geq n_{0}$, then $\phi_{3}$ vanishes identically on $V_{n_{0}}$ (Hurwitz' theorem) and so in $\mathcal{N}$. However, from a) we infer that $\left\|Y-Y_{1}\right\| \leq \epsilon \sum_{n=1}^{\infty} 1 / n^{2}=\epsilon \pi^{2} / 6$ and so the third coordinate of $Y$ is non-constant provided that $\epsilon$ is small enough, a contradiction. 
Set $h: \mathcal{N} \rightarrow \mathbb{R}$ by $h(P)=\operatorname{Re} \int_{P_{0}}^{P} \phi_{3}$, where $P_{0}$ is an arbitrary fixed point in $\mathcal{N}$. Applying Theorem 4.1 to $h$ and $p$ we obtain a complete conformal minimal immersion $X=\left(X_{1}, X_{2}, X_{3}\right): \mathcal{N} \rightarrow \mathbb{R}^{3}$ such that $p_{X}=p$ and $X_{3}=h$. As $\partial X_{3}=\phi_{3}$ never vanishes on $\mathcal{N}$ then the meromorphic Gauss map of $X$ has neither zeros nor poles, concluding the proof.

Open Riemann surfaces carrying non-constant bounded harmonic functions are hyperbolic, but the reciprocal is false in general. However, in the case of finite topology both statements are equivalent. Even more, if $\mathcal{N}$ is biholomorphic to a compact Riemann surface minus a finite collection of at least two pairwise disjoint closed discs, then there exists proper harmonic maps $h: \mathcal{N} \rightarrow(0,1)$. As a consequence,

Corollary 4.3. Any of the following statements holds:

(a) $\mathcal{N}$ carries a non-constant bounded harmonic function if and only if there exists a conformal complete non-flat minimal immersion of $\mathcal{N}$ in a horizontal slab of $\mathbb{R}^{3}$.

(b) If $\mathcal{N}$ is hyperbolic and of finite topology, then there exists a conformal complete non-flat minimal immersion of $\mathcal{N}$ in a horizontal slab of $\mathbb{R}^{3}$.

(c) If $\mathcal{N}$ is biholomorphic to a compact Riemann surface minus a finite collection of at least two pairwise disjoint closed discs, then $\mathcal{N}$ admits a proper conformal complete non-flat minimal immersion in an open horizontal slab of $\mathbb{R}^{3}$.

In addition, in any case the first two coordinates of the flux map can be prescribed.

If $h$ is the real part of a non-constant holomorphic function and $p=0$, Theorem 4.1 also gives that:

Corollary 4.4. Any of the following statements holds:

(d) The following assertions are equivalent:

- $\mathcal{N}$ carries a non-constant bounded holomorphic function.

- There exists a full* complete null immersion of $\mathcal{N}$ in $\mathbb{C}^{2} \times \mathbb{D}$.

- There exists a full complete holomorphic immersion of $\mathcal{N}$ in $\mathbb{C} \times \mathbb{D}$.

(e) If $\mathcal{N}$ is hyperbolic and of finite topology, then there exists a full complete null immersion of $\mathcal{N}$ in $\mathbb{C}^{2} \times \mathbb{D}$ and a full complete holomorphic immersion of $\mathcal{N}$ in $\mathbb{C} \times \mathbb{D}$.

(f) If $\mathcal{N}$ admits a proper holomorphic function into the unit disk, then $\mathcal{N}$ admits a full proper complete minimal immersion in $\mathbb{C}^{2} \times D$ and a full proper complete holomorphic immersion in $\mathbb{C} \times D$, where $D$ is any simply connected planar domain (the case $D=\mathbb{C}$ is proved in $[\mathrm{AL}]$ ).

\footnotetext{
${ }^{*}$ A complex curve in $\mathbb{C}^{n}$ is said to be full if it is not contained in a linear complex subspace.
} 
Remark 4.5. The family of Riemann surfaces involved in item (d) (and so in item (a)) contains examples with any open orientable topological type.

The family of Riemann surfaces concerning item (f) is also very vast. For instance, it includes all the finitely sheeted ramified coverings of the unit disc.

Although the first statement of the above remark is well known, for completeness we sketch a proof based on Scheinberg approximation results [Sc]. Let $\mathcal{N}$ be an open Riemann surface, and consider two compact regions $M, V \subset \mathcal{N}$ such that $M \subset V^{\circ}$, $\chi\left(V^{\circ}-M\right)=-1$ and $V^{\circ}-M$ has no bounded components in $V^{\circ}$. Take also $\epsilon>0$ and a non-constant holomorphic function $f: M \rightarrow \mathbb{D}$. Consider a Jordan $\operatorname{arc} \gamma \subset V^{\circ}-M$ with endpoints in $\partial(M)$ and otherwise disjoint from $\partial(M)$ such that $\chi\left(V^{\circ}-(M \cup \gamma)\right)=0$ and $V^{\circ}-(M \cup \gamma)$ has no bounded components in $V^{\circ}$. For simplicity write $S=M \cup \gamma$. Construct a continuous function $\hat{f}: S \rightarrow \mathbb{D}$ with $\left.\hat{f}\right|_{M}=f$, and use Scheinberg approximation theorem to find a compact tubular neighborhood $\tilde{M}$ of $S$ in $V^{\circ}$ and a holomorphic function $\tilde{f}: \tilde{M} \rightarrow \mathbb{D}$ such that $\chi\left(V^{\circ}-\tilde{M}\right)=0$ and $\|\tilde{f}-f\|<\epsilon$ on $M$. Applying recursively this argument, we can find sequences $\left\{V_{n}\right\}_{n \in \mathbb{N}}$ of compact regions in $\mathcal{N}$ and holomorphic functions $\left\{f_{n}: V_{n} \rightarrow \mathbb{D}\right\}_{n \in \mathbb{N}}$, such that:

- $V_{n} \subset V_{n+1}^{\circ}, \chi\left(V_{n+1}^{\circ}-V_{n}\right)=-1, V_{n+1}^{\circ}-V_{n}$ has no bounded components in $V_{n+1}^{\circ}$ and $N:=\bigcup_{n \in \mathbb{N}} V_{n}$ is homeomorphic to $\mathcal{N}$, and

- $\left\|f_{n+1}-f_{n}\right\|<\epsilon 2^{-n-1}$ on $V_{n}$ for all $n$, where $\epsilon=\max _{V_{1}}\left|f_{1}\right|-\min _{V_{1}}\left|f_{1}\right|>0$.

The sequence $\left\{f_{n}\right\}_{n \in \mathbb{N}}$ converges uniformly on compact subsets of $N$ to a nonconstant bounded holomorphic function $u: N \rightarrow \mathbb{C}$. The proof is done.

We finish by proving a Lorentzian version of Theorem 4.1 for weakly complete maximal surfaces in the Lorentz-Minkowski 3-spacetime $\mathbb{R}_{1}^{3}$ with signature $(-,+,+)$. Recall that a conformal maximal immersion $X: M \rightarrow \mathbb{R}_{1}^{3}$ with singularities is said to be weakly complete if the metric $\sum_{j=1}^{3}\left|\phi_{j}\right|^{2}$ is complete on $M$, where $\Phi=\left(\phi_{1}, \phi_{2}, \phi_{3}\right)$ are the Weierstrass data of $X$ (see [UY]).

Corollary 4.6. Let $h: \mathcal{N} \rightarrow \mathbb{R}$ be a non-constant harmonic function.

Then there exist weakly complete conformal maximal immersions

$$
Y=\left(Y_{1}, Y_{2}, Y_{3}\right): \mathcal{N} \rightarrow \mathbb{R}_{1}^{3}
$$

and $Z=\left(Z_{1}, Z_{2}, Z_{3}\right): \mathcal{N} \rightarrow \mathbb{R}_{1}^{3}$ with $Y_{1}=h=Z_{2}$.

Proof. Let $X=\left(X_{1}, X_{2}, X_{3}\right): \mathcal{N} \rightarrow \mathbb{R}^{3}$ be the immersion in Theorem 4.1 associated to $h$ and the group morphism $p: \mathscr{H}_{1}(\mathcal{N}, \mathbb{Z}) \rightarrow \mathbb{R}^{3}, p(\gamma)=\left(0,0, \operatorname{Im} \int_{\gamma} \partial h\right)$ for all $\gamma \in \mathscr{H}_{1}(\mathcal{N}, \mathbb{Z})$. Labeling $X_{j}^{*}$ as the conjugate harmonic function of $X_{j}, j=1,2$, then $Y=\left(X_{3}, X_{2}^{*}, X_{1}^{*}\right): \mathcal{N} \rightarrow \mathbb{R}_{1}^{3}$ and $Z=\left(X_{1}^{*}, X_{3}, X_{2}\right): \mathcal{N} \rightarrow \mathbb{R}_{1}^{3}$ satisfy the conclusion of the corollary. 


\section{References}

[AF] A. Alarcón and I. Fernández, Complete minimal surfaces in $\mathbb{R}^{3}$ with a prescribed coordinate function. Differential Geom. Appl. 29 (2011), suppl. 1, S9-S15.Zbl 1230.53012 MR 2831994

[AFL] A. Alarcón, I. Fernández and F. J. López, Complete nonorientable minimal surfaces and harmonic funtions. In preparation.

[AFM] A. Alarcón, L. Ferrer and F. Martín, Density theorems for complete minimal surfaces in $\mathbb{R}^{3}$. Geom. Funct. Anal. 18 (2008), 1-49. Zbl 1144.53013 MR 2399094

[AL] A. Alarcón and F. J. López, Minimal surfaces in $\mathbb{R}^{3}$ properly projecting into $\mathbb{R}^{2}$. J. Differential Geom. 90 (2012), 351-381. Zbl 06053706

[AL2] A. Alarcón and F. J. López, Null curves in $\mathbb{C}^{3}$ and Calabi-Yau conjectures. Math. Ann., to appear; preprint, arXiv:0912.2847 [math.DG].

[CM] T. H. Colding and W. P. Minicozzi II, The Calabi-Yau conjectures for embedded surfaces. Ann. of Math. (2) 167 (2008), 211-243. Zbl 1142.53012 MR 2373154

[FMM] L. Ferrer, F. Martín and W. H. Meeks III, Existence of proper minimal surfaces of arbitrary topological type. Adv. Math. 231 (2012), 378-413. Zbl 06060990 MR 2935393

[Fu] H. Fujimoto, On the number of exceptional values of the Gauss maps of minimal surfaces. J. Math. Soc. Japan 40 (1988), 235-247. Zbl 0629.53011 MR 0930599

[JX] L. P. M. Jorge and F. Xavier, A complete minimal surface in $\mathbb{R}^{3}$ between two parallel planes. Ann. of Math. (2) 112 (1980), 203-206. Zbl 0455.53004 MR 0584079

[Lo1] F. J. López, A nonorientable complete minimal surface in $\mathbb{R}^{3}$ between two parallel planes. Proc. Amer. Math. Soc. 103 (1988), 913-917. Zbl 0662.53008 MR 0947681

[Lo2] F. J. López, Hyperbolic complete minimal surfaces with arbitrary topology. Trans. Amer. Math. Soc. 350 (1998), 1977-1990. Zbl 0901.53005 MR 1422904

[Lo3] F. J. López, Uniform approximation by complete minimal surfaces of finite total curvature in $\mathbb{R}^{3}$. Trans. Amer. Math. Soc., to appear; preprint arXiv:0903.3209v7 [math.DG].

[MM] F. Martín and S. Morales, Complete proper minimal surfaces in convex bodies of $\mathbb{R}^{3}$. Duke Math. J. 128 (2005), 559-593. Zbl 1082.53009 MR 2145744

[Na] N. Nadirashvili, Hadamard's and Calabi-Yau's conjectures on negatively curved and minimal surfaces. Invent. Math. 126 (1996), 457-465. Zbl 0881.53053 MR 1419004

[Os1] R. Osserman, Minimal surfaces in the large. Comment. Math. Helv. 35 (1961), 65-76. Zbl 0098.34904 MR 0123248

[Os2] R. Osserman, A survey of minimal surfaces. Second edition, Dover Publications, New York 1986. Zbl 0209.52901 MR 0852409

[RT] H. Rosenberg and E. Toubiana, A cylindrical type complete minimal surface in a slab of $\mathbb{R}^{3}$. Bull. Sci. Math. (2) 111 (1987), 241-245. Zbl 0631.53012 MR 0912952

[Sc] S. Scheinberg, Uniform approximation by functions analytic on a Riemann surface. Ann. of Math. (2) 108 (1978), 257-298. Zbl 0423.30035 MR 0499183

[UY] M. Umehara and K. Yamada, Maximal surfaces with singularities in Minkowski space. Hokkaido Math. J. 35 (2006), 13-40. Zbl 1109.53016 MR 2225080 
[Xa] F. Xavier, The Gauss map of a complete nonflat minimal surface cannot omit 7 points of the sphere. Ann. of Math. (2) 113 (1981), 211-214. Zbl 0477.53007 MR 0657243

Received October 22, 2009

Antonio Alarcón, Departamento de Geometría y Topología, Universidad de Granada, 18071 Granada, Spain

E-mail: alarcon@ugr.es

Isabel Fernández, Departamento de Matemática Aplicada I, Universidad de Sevilla, 41012 Sevilla, Spain

E-mail: isafer@us.es

Francisco J. López, Departamento de Geometría y Topología, Universidad de Granada, 18071 Granada, Spain

E-mail: fjlopez@ugr.es 